\title{
Selected Topics on Requirements Engineering: Editorial Introduction to the Issue 3 of CSIMQ
}

\author{
Peter Forbrig \\ University of Rostock, Institute of Computer Science, \\ Chair in Software Engineering, \\ Albert-Einstein-Str. 22, 18051 Rostock, Germany \\ peter.forbrig@uni-rostock.de
}

For this issue we collected five papers that fall into the domain of Requirements Engineering. It is well known that wrong requirements cause a lot of problems. Some projects totally fail because of that, others waste a lot of money because the correction of resulted errors in the implementation is very time consuming and labour intensive. Therefore, new ideas in identifying the correct requirements are very important. In the first edition of the journal of Requirements Engineering in 2015 Leah Goldin and Daniel M. Berry [1] discuss the question whether in the development of large scale systems the institutionalized, proactive requirements reuse pays off. In their case study they found out that at least for the studied project it paid off to meet the moving target of requirements based on existing specifications. Reuse of requirements specification might be one way to reduce time to market. However, there are still a lot of aspects to consider like the kind of specification languages for functional and non-function requirements. Security might be one of the most important aspects that prevent users from applying software systems.

Christoph Oemig discusses the idea of pre-sales requirements engineering in his paper "When Analysts turn into Boxers: An Introduction to Pre-Sales Requirements Engineering". In the presales phase the major goal is to provide customers compelling offers that exactly fit their needs. The author uses the metaphor of boxers for analysts. He argues with terms like fighter, coach, opponent, and referee for roles and terms like training, fight, and analysis for phases. One can also "throw the towel" in requirements engineering. This discussion of analogies might draw a lot of interest on his paper, which is the first one in Issue 3 of CSIMQ.

Elaborated system models, enterprise architectures, ontologies, and information logistics representations are the basis of current engineering-based approaches. They are designed and mainly applied for large enterprises that have relatively long change cycles for their systems. If such changes have to be performed more frequently a much higher flexibility is required. There is a need for agile engineering that requires continuous requirements engineering (CRE). CRE can only be successful if it combines rigid engineering principles with agility, emergence, and spontaneity to support sustainability and viability of the systems under development. During the 21st Working Conference on Requirements Engineering: Foundation for Software Engineering 2015 (REFSQ 2015) in Essen the 1st Workshop on Continuous Requirements Engineering (CRE'15) was organized. The proceedings can be found in [2]. Three extended versions of the workshop's papers are published in this issue of CSIMQ. They are entitled "Getting Grip on Security Requirements Elicitation by Structuring and Reusing Security Requirements Sources", "Dynamic Socio-technical System Design based on Stakeholder 
Interaction. A Programming while Modeling Approach", and "Conceptual Model of User Adaptive Enterprise Application".

Christian Schmidt and Peter Liggesmeyer focus on requirements for security. They discuss their approach by structuring and reusing resources. Advantages and disadvantages of the provided methodology are as well discussed from an academic and industrial points of view.

Security aspects are discussed also in the paper "A Model-driven Role-based Access Control for SQL Databases" written by Raimundas Matulevicius and Henri Lakk. They focus on information systems and follow a model-driven approach. Their Role-based access control is specified in SecureUML, an extension of UML by stereotypes, tagged values and constraints. The model of role-based access control is transformed to security constraints on database views. The transformation process is controlled by a set of templates that are considered as transformation rules.

Some identified requirements might have been correct during the initial phase of a project. But they might have become outdated during implementing the software or even later during usage of the application. It is very important to have strategies that allow the update of requirements and an easy adaptation of the corresponding software.

In their paper: "Dynamic Socio-technical System Design based on Stakeholder Interaction" Albert Fleischmann, Werner Schmidt and Chris Stary assume that modelling might help to allow end users to adapt running systems. They focus on stakeholder interaction and subject-oriented modelling of business processes. The language they use is called S-BPM. It is supported by certain interpreters that allow the direct execution of specifications. It is suggested that end users are able to manipulate specifications and in this way adapt an application to changing requirements. This is even possible during runtime.

Finally, there is the paper of Inese Supulniece and Janis Grabis "Conceptual Model of User Adaptive Enterprise Application" that follows the same idea of user adaptation as the previous one. However, they focus on enterprise applications and on using Meta-Models for describing possible user adaptations. They call systems designed in this way User Adaptive Enterprise Applications (UAEA) and they argue that user adaptation has to be a goal from the very beginning of the system development. It is difficult to integrate it later.

In this issue there are shown five different views on solutions in requirements engineering. They present interesting ideas that might influence further research on continuous requirements engineering. I am sure that readers can pick up some of these ideas for their own projects and research.

\section{References}

[1] L. Goldin, D. M. Berry, "Reuse of requirements reduced time to market at one industrial shop: a case study," Requirements Engineering, Springer, vol. 20, Issue 1, pp. 23-44, 2015. http://dx.doi.org/10.1007/s00766-0130182-7

[2] Joint Proceedings of REFSQ-2015 Workshops, Research Method Track, and Poster Track, co-located with the 21st International Conference on Requirements Engineering: Foundation for Software Quality (REFSQ 2015), Essen, DE, 2015. Available: http://ceur-ws.org/Vol-1342/ (accessed July 9th 2015) 